\title{
An Overview on the Facile and Reversible Cations Intercalation in Nickel-Hexacyanoferrate Open Framework
}

\author{
Michela Ciabocco ${ }^{1}$, Mario Berrettoni ${ }^{1,}{ }^{*}$, Silvia Zamponi ${ }^{2}$, Reno Spinosi $^{2}$ and Paolo Conti ${ }^{2}$ \\ ${ }^{1}$ Dipartimento di Chimica Industriale “Toso Montanari”, UOS, Campus di Rimini, Università di \\ Bologna, Rimini, Italy \\ ${ }^{2}$ Scuola di Scienze e Tecnologie, Università di Camerino, Camerino, Italy \\ *E-mail: mario.berrettoni@ unibo.it
}

doi: $10.20964 / 2018.06 .37$

Received: 14 February 2018 / Accepted: 23 March 2018 / Published: 10 May 2018

\begin{abstract}
The paper reports a detailed study about the influence of the electrolyte composition in nickelhexacyanoferrate (NiHCF) electrochemical behavior. Shapes and positions of the CV waves are strongly related to the cation of the supporting electrolyte, which undergoes intercalation/deintercalation during the redox process. The electrochemical behavior of NiHCF has been characterized in different electrolyte solutions containing either pure monovalent or trivalent metal nitrates. Nickelhexacyanoferrate permits the reversible insertion of a wide variety of monovalent, divalent and trivalent ions in aqueous solution; the characteristic potential $E_{\mathrm{p}}$ of the cathodic process depends upon the nature of the intercalated cation. Electrochemical measurements demonstrate the unprecedented fast kinetics of trivalent ion insertion associated with this material. The reported findings represent the first systematic step toward understanding the principles of multivalent charge screening and provide a novel cation intercalation mechanism in $\mathrm{NiHCF}$, assisted by both water molecules and ferrocyanide vacancies. The synergistic mechanism can reduce the electrostatic repulsion and provide a preferential path for the rapid cation intercalation.
\end{abstract}

Keywords: alkali cations, cation exchange, ion sensing, nickel-hexacyanoferrate, trivalent cations.

\section{$\underline{\text { FULL TEXT }}$}

(C) 2018 The Authors. Published by ESG (www.electrochemsci.org). This article is an open access article distributed under the terms and conditions of the Creative Commons Attribution license (http://creativecommons.org/licenses/by/4.0/). 